\title{
Viscoelastic instabilities in micro-scale flows
}

Francisco J. Galindo-Rosales ${ }^{\mathrm{a}}$, Laura Campo-Deaño ${ }^{\mathrm{a}}$, Patrícia C. Sousa ${ }^{\mathrm{a}}$, Vera M. Ribeiro ${ }^{a}$, Mónica S. N. Oliveira ${ }^{\mathrm{b}}$, Manuel A. Alves ${ }^{\mathrm{a}}$, Fernando T. Pinho $^{\mathrm{a}, *}$

${ }^{a}$ CEFT, Faculdade de Engenharia da Universidade do Porto, Rua Dr. Roberto Frias, 4200-465 Porto, Portugal

${ }^{b}$ James Weir Fluids Laboratory, Department of Mechanical and Aerospace Engineering, University of Strathclyde, Glasgow G1 1XJ, UK

\begin{abstract}
Many artificial and natural fluids contain macromolecules, particles or droplets that impart complex flow behaviour to the fluid. This complex behaviour results in a non-linear relationship between stress and deformation standing in between Newton's law of viscosity for an ideal viscous liquid and Hooke's law for an ideal elastic material. Such non-linear viscoelastic behaviour breaks down flow reversibility under creeping flow conditions, as encountered at the micro-scale, and can lead to flow instabilities. These instabilities offer an alternative to the development of systems requiring unstable flows under conditions where chaotic advection is unfeasible. Flows of viscoelastic fluids are characterized by the Weissenberg $(W i)$ and Reynolds $(R e)$ numbers, and at the micro-scale flow instabilities occur in regions in the $W i$-Re space typically unreachable at the macro-scale, namely high $W i$ and low $R e$. In this paper, we review recent experimental work by the authors on the topic of elastic instabilities in flows having a strong extensional component, includ-
\end{abstract}

\footnotetext{
${ }^{*}$ Corresponding author. Tel:+ 35225081597

Email address: fpinho@fe.up.pt (Fernando T. Pinho )

Preprint submitted to Experimental Thermal and Fluid Science

March 10, 2014
} 
ing: flow through a hyperbolic contraction followed by a sudden expansion; flow in a microfluidic diode and in a flow focusing device; flow around a confined cylinder; flow through porous media and simplified porous media analogues. These flows exhibit different types of flow transitions depending on geometry, $W i$ and $R e$, including: transition from a steady symmetric to a steady asymmetric flow, often followed by a second transition to unsteady flow at high $W i$; direct transition between steady symmetric and unsteady flows.

Keywords: Viscoelasticity, Elastic instabilities, Microfluidics

\section{Introduction}

2 The use of microfluidic devices is growing fast in a variety of applications 3 in biochemistry, drug delivery, medical diagnosis, micro-heat exchangers, se4 quencing and synthesis of chemicals, micro-mixing or micro-rheology, among 5 others [1]. Most of the research in this field concerns Newtonian fluids [2] 6 with their flows characterized by linear behaviour typical of low Reynolds 7 number flows, although chaotic advection can also occur in laminar flows 8 at sufficiently high Reynolds numbers [3]. However, a significant number of 9 fluids (natural and mostly synthetic) includes macromolecules, particles or 10 droplets that impart complex properties to the fluids, such as viscoelasticity, 12 fluids [4].

13 The Reynolds number $(R e)$ is defined as the ratio between inertial and vis14 cous forces, $R e=\rho U L / \eta$, and often requires the definition of a characteristic 15 rate of deformation $\dot{\gamma} \approx U / L$ from relevant velocity $(U)$ and length scales $(L)$ 
in order to determine the characteristic shear viscosity $\eta$ for non-Newtonian fluids. The ratio between the relaxation time of the fluid $(\lambda)$ and a characteristic time scale of the flow $\left(t_{\text {flow }}=L / U\right)$ is the so-called Deborah number $(D e)$ given by $D e=\lambda U / L$, which is a dimensionless measure of the rate of change of flow conditions related to flow unsteadiness in a Lagrangian perspective [5]. In shear flows, viscoelastic fluids are also subject to shear-driven normal stresses and the ratio between the normal and shear stresses quantifies the non-linear response of the viscoelastic fluid and is proportional to the Weissenberg number $(W i=\lambda \dot{\gamma})$, so that the final form of the Weissenberg number in many flows looks like that of the Deborah number, but it has a clearly different physical meaning (and in some cases may also involve a different length scale when normal stresses and Lagrangian transients co-exist) $[5,6]$.

The small length scales in microfluidics increases significantly the relevance of fluid elasticity and allows exploring regions of the $W i$-Re parameter space typically unreachable at the macro-scale, i.e. low $R e$ and high $W i$, represented by large values of the Elasticity number $\left(E l=W i / R e=\lambda \eta / \rho L^{2}\right)[7]$. Therefore, at the micro-scale, flows of viscoelastic fluids can be significantly different from those of their Newtonian counterparts, because elastic instabilities can be triggered with relative ease. One illustrative example of this phenomenon can be found in the human circulatory system, as there is recent evidence of viscoelastic behaviour of human blood and plasma, which is enhanced in the microcirculation $[8,9,10]$. Purely elastic flow instabilities in shear flow have been extensively studied both at macro [11] and micro-scales [12] and it is widely accepted that the underlying mechanism is related to the 
elastic normal stresses developing along curved streamlines being unable to sustain minor perturbations appearing on those streamlines $[13,14,15,16]$. Pakdel and McKinley $[14,15,17]$ showed that the critical conditions for the onset of purely elastic instabilities can be described for a wide range of flows by a single dimensionless parameter $(M)$, which accounts for elastic normal stresses and streamline curvature:

$$
M=\sqrt{\frac{\lambda v}{\Re} \frac{\tau_{11}}{\tau_{12}}},
$$

${ }_{47}$ where $\lambda$ is the relaxation time of the fluid, $v$ is the local streamwise fluid 48 velocity, $\tau_{11}$ is the local tensile stress in the flow direction, $\tau_{12}$ is the shear

stress $\left(\tau_{12}=\eta \dot{\gamma}\right)$ and $\Re$ is the streamline local radius of curvature. When the flow conditions are such that $M$ locally exceeds a critical value, $M_{\text {crit }}$, elastic instabilities develop. The value of $M_{\text {crit }}$ is slightly dependent on the flow, and for simple flows, where the radius of curvature is known, $M_{\text {crit }}$ can be estimated. As discussed by McKinley et al. [15], for Taylor-Couette flow $M_{\text {crit }} \approx 5.9$ and for torsional flow in a cone-and-plate arrangement, $M_{\text {crit }} \approx 4.6$. For more complex flows (see example in Figure 1), the spatial variation of $M$ needs to be taken into account to identify the critical regions where the largest value of $M$ occurs. This mechanism for the onset of purely elastic instabilities and the applicability of the $M$ parameter to identify the critical conditions for the onset of elastic instabilities was confirmed numerically by Alves and Poole [18] for creeping flow of upper-convected Maxwell (UCM) fluids in smooth contractions, for a wide range of contraction ratios.

The experimental and numerical studies of purely elastic flow instabilities 
developing in several micro-geometries for extensional-dominated flows have essentially emerged in the last decade (e.g. [12, 19, 20]). Among these arrangements, viscoelastic flow in contraction geometries has been the subject of numerous investigations. Although the instability onset can be linked to the ubiquitous presence of large normal stresses, and streamline curvature is also present, in other micro-geometries able to generate extensional dominated flows (e.g. stagnation/ flow focusing devices) or mixed kinematic flows (e.g. contraction/expansions), a clear picture of all the observed transitions and their causes has not yet emerged [21]. These are conditions that justify our research program exploring various flows possessing a strong extensional deformation flow field in order to identify common features. These flows exhibit a rich variety of unexpected effects, usually anchored on elastic effects. The high sensitivity of viscoelastic fluid flow at the micro-scale to flow instabilities under creeping flow conditions offer an alternative to the development of systems requiring unstable flows under conditions where chaotic advection is impossible or difficult to achieve, while they also impose limits of operation for systems where instabilities are to be avoided at all, as in micro-rheology. The next section briefly describes the experimental techniques prior to the presentation and discussion of experimental results obtained in five different geometrical arrangements, where the common thread is the presence of a strong extensional flow component and the onset of elastic instabilities at sufficiently high $W i$. 


\section{Materials and methods}

The viscoelastic fluids typically used in our works consist of aqueous polymer solutions with a high molecular weight, namely polyacrylamide (PAA, $M_{w}=18 \times 10^{6} \mathrm{~g} \mathrm{~mol}^{-1}$, Polysciences) or polyethylene oxide (PEO, $M_{w}=8 \times 10^{6}$ g mol ${ }^{-1}$, Sigma-Aldrich), at different weight concentrations (see Table 1 for details).

All these fluids were characterized rheologically under simple shear flow by means of a rotational rheometer (Physica MCR301, Anton Paar) to determine the dependence of the shear viscosity on the shear rate (Figure 2a). In this experiment the first normal stress difference $\left(N_{1}\right)$ can also be determined from the measured normal force. However, measuring $N_{1}$ in dilute and semidilute polymer solutions is rather difficult and obtaining reproducibility in the results is challenging. In these cases, it is more effective to perform the characterization under uniaxial elongational flow as in a capillary-breakup extensional rheometer (Haake CaBER1, Thermo Scientific), which measures the time evolution of the diameter of a stretched fluid filament as it thins, from which the longest relaxation time of the fluids can be determined (Figure 2b) [22]. Despite the significant differences between a steady shear flow (as in a shear rheometer) and an unsteady extensional flow (as in the CaBER device), there is a direct relationship between the elastic properties measured in both devices. Indeed, Zell et al. [23] have found that the normal stress coefficient, measured in shear flow and defined as $\Psi_{10}=\frac{N_{1}}{\dot{\gamma}^{2}}$, shows a quadratic dependence on the relaxation time determined in the CaBER. Since the normal stress difference coefficient depends on a shear relaxation time, the relation between the shear and extensional relaxation times may 
seem obvious but there are different dependences that remain unexplained as discussed by Zell et al. [23].

The viscoelastic fluids used in the experiments usually exhibit a shear thinning behaviour, i.e. their apparent shear viscosity decreases with the increase in the applied shear rate. Moreover, the higher the molecular weight and the concentration of the polymer additive, the higher the shear viscosity, the stronger the shear thinning behaviour and the longer the relaxation time will be $[24,25]$. These effects are clearly shown in Figure 2, where the viscosity of the various fluids is plotted as a function of the shear rate in Figure $2 \mathrm{a}$ and the time evolution of the normalized diameters measured in the CaBER device are shown in Figure 2b. A small percentage of glycerol was added to one of the fluids, in order to increase slightly the viscosity of the solution increasing in turn the relaxation time of the polymer solution. Typically, Boger fluids are prepared by dissolving a flexible polymer with large molecular weight in a high viscosity solvent, to minimize the shear thinning due to the addition of the polymer. However, the use of high-viscosity fluids in microfluidics is precluded by the large pressure drops their flows develop with severe consequences to the structural integrity of the chips. The advent of Boger fluids with low viscosity is a useful alternative for microfluidics when it is important to distinguish between shear thinning and elastic effects. By adding salt to aqueous solutions of polyacrylamide, Aitkadi et al. [26] found that the salt has a stabilizing effect on the shear viscosity while maintaining adequate levels of elasticity, thus resulting in a Boger fluid behaviour, a good example being the PAAsg200 fluid shown in Figure 2. 
No storage or loss moduli $\left(G^{\prime}\right.$ and $\left.G^{\prime \prime}\right)$ are presented because it was not possible to measure $G^{\prime}$ accurately in small amplitude oscillatory shear (SAOS) in this rotational rheometer due to instrument limitations and the low elasticity of the fluids used. All the rheological experiments, as well as all the microfluidic experiments, were performed at a constant temperature of $20^{\circ} \mathrm{C}$.

The geometries used were planar micro-channels made of polydimethylsiloxane (PDMS) fabricated from SU-8 photoresist moulds using standard softlithography techniques, as described in [27, 28]. The micro-fabrication process consists of four fundamental steps: 1) drawing of the micro-geometries using a CAD software; 2) manufacture of the mask using the CAD drawings - this type of mask is obtained by sputtering a chrome layer onto a glass or quartz substrate in which the CAD image is etched; 3) manufacture of the SU-8 mould by photolithography using the mask; and 4) fabrication of the PDMS micro-channels using the mould. High-resolution chrome masks were employed to obtain high quality SU-8 moulds with nearly vertical side-walls and well-defined corner features. The SU-8 photoresist was used to create a positive-relief on the mould surface, producing a mould containing the inverse structure of the micro-channels. The microfluidic devices were then fabricated by casting PDMS on the mould. The PDMS used (Sylgard 184, Dow Corning) is commercially available as a prepolymer kit composed of a PDMS oligomer and a crosslinking agent or curing agent, which are mixed in certain proportions (typically 50:1, 20:1 and 10:1 PDMS:curing agent) to produce the polymer used to fabricate the micro-devices. An overview of the micro-channel fabrication procedure is shown schematically in Figure 3. In 
the next section the different geometrical arrangements and their influence on the fluid flow will be described in detail. Figure 4 shows scanning electron microscopy (SEM) pictures of the micro-channels used and Table 2 lists the corresponding dimensions.

Pressure measurements were performed using differential pressure transducers (Honeywell 26 PC sensors) operating up to a maximum differential pressure of $210 \mathrm{kPa}$. Pressure transducers with different ranges and sensitivities were used depending on the fluids and flow rates tested. The flow rate was always imposed using a syringe pump, such as the neMESYS (Cetoni GmbH) and PHD 2000 (Harvard Apparatus), equipped with different Hamilton syringes (from $25 \mu \mathrm{l}$ to $1 \mathrm{ml}$, depending on the required flow rate) to ensure pulsation-free dosing.

Flow visualizations were based on streak photography with the micro-channels placed on the motorized stage of an inverted epi-fluorescence microscope (DMI 5000M, Leica Microsystems GmbH) equipped with a sensitive monochromatic CCD camera (DFC350 FX, Leica Microsystems GmbH). The fluids were seeded with fluorescent tracer particles (1 $\mu \mathrm{m}$ diameter Nile Red particles, Molecular Probes, Invitrogen, Ex/Em: 520/580 nm) and the illumination was provided by a $100 \mathrm{~W}$ mercury lamp operating together with adequate excitation and emission filters and a dichroic mirror.

For the velocity measurements, a micro-Particle Image Velocimetry ( $\mu$ PIV) system from Dantec Dynamics was used. Images of the emitted radiation from excited fluorescent tracer particles were captured using a $20 \times$ (numerical aperture, $\mathrm{NA}=0.4$ ) microscope objective and a camera (Flow Sense 4M, Dantec Dynamics), with a resolution of $2048 \times 2048$ pixels and running on 
double frame mode. The flow was illuminated by a double-pulsed $532 \mathrm{~nm}$ Nd:YAG laser (Dual Power 65-15, Dantec Dynamics). The fluorescent particles used in $\mu \mathrm{PIV}$ were smaller than those used in streak photography and had a diameter of $0.5 \mu \mathrm{m}$.

\section{Results and Discussion}

In this section, we review five different experimental investigations in extensionaldominated flows of viscoelastic fluids which explore the onset of elastic instabilities. The case studies focus on fundamental issues to understand the flow dynamics itself, but also cover more applied research ranging from fluid characterization in a microfluidic rheometer to the development of a microfluidic diode, which can be used as a flow rectifier or in micro-pumps.

\subsection{Microfluidic hyperbolic contraction and sudden expansion}

The flow of Boger fluids in hyperbolic contraction micro-channels was investigated to assess the relation between the observed flow patterns and the dimensionless relaxation time of the fluids. The underlying rationale for using hyperbolic-shaped channels is their ability to generate strong extensional flows with enhanced strain-rate homogeneity near the centreline when compared to other contraction flows $[29,30]$. This geometry is thus sensitive to effects of elongational viscosity and the use of Boger fluids allows a clear separation between elastic and viscous effects [31].

In their investigation on the flow through hyperbolic contraction and sudden expansion, Campo-Deaño et al. [32] used aqueous solutions of PAA at different weight concentrations $(50,125,250$ and $400 \mathrm{ppm})$ to which $1 \%$ of $\mathrm{NaCl}$ were added (Table 1). The shear viscosity of the fluids ranges from 1 to 4 
$\mathrm{mPa} \cdot \mathrm{s}$ (Figure 2).

The geometry imaged in Figure 4a) includes a hyperbolic contraction followed by a sudden expansion. The width of the inlet and outlet channels is $D_{1}=400 \mu \mathrm{m}$, the minimum width of the contraction is $D_{2}=54 \mu \mathrm{m}$ and the length of the hyperbolic contraction region is $L_{c}=128 \mu \mathrm{m}$, resulting in a total Hencky strain of $\varepsilon_{H}=\ln \left(D_{1} / D_{2}\right)=2$. The depth of the micro-channel is constant, $h=45 \mu \mathrm{m}$.

Visualizations of the flow patterns in the hyperbolic contraction/abrupt expansion micro-channel (Figure 5) showed a Newtonian-like behaviour at very low flow rates (low $W i$ ) and complex non-Newtonian behaviour at high flow rates (high $W i$ ) for all polymer concentrations. This non-Newtonian behaviour corresponds to the existence of regions of separated flow upstream of the contraction. Thus, for negligible inertia and elasticity no flow separation was observed. For the Boger fluid with $400 \mathrm{ppm}$ of PAA and $1 \% \mathrm{NaCl}$ the critical flow rate at which the behaviour first changes from Newtonian-like to non-Newtonian was determined as $Q_{c r}=0.17 \mathrm{ml} / \mathrm{hr}$, which corresponds to $R e=0.7$ and $W i=4$; for the PAA solution at $250 \mathrm{ppm}$ the critical value is $Q_{c r}=0.27 \mathrm{ml} / \mathrm{hr}(R e=1.2, W i=4) ;$ for $125 \mathrm{ppm} Q_{c r}=0.47 \mathrm{ml} / \mathrm{hr}(R e=$ 2.8, $W i=3.6$ ), and finally for the lowest concentration of $50 \mathrm{ppm}$ the critical flow rate corresponds to $Q_{c r}=0.95 \mathrm{ml} / \mathrm{hr}(R e=5.8, W i=2.8)$. Figure 5 shows flow patterns above this critical flow rate for each case. As expected, there is a clear decrease in the critical flow rate when the polymer concentration is increased due to enhanced elasticity with the corresponding increase of the relaxation time. However, the transition was found to correspond to a critical value of $W i$ of about 4 , which did not decrease significantly except 
when flow inertia became relevant $(R e>2)$. Above the critical flow rate, symmetric vortices develop upstream of the hyperbolic contraction in contrast to the behaviour of Newtonian liquids that exhibit vortices downstream as a consequence of inertial effects. Further increasing the Weissenberg number leads to an increase of the upstream vortex due to the enhancement of elastic effects which is characterized in terms of the dimensionless vortex length, $X_{R}=L_{v} / D_{1}$, where $L_{v}$ is the vortex length. This variation of $X_{R}$ with $W i$ is shown in Figure 6 and it can be used as a simple methodology to estimate the value of the relaxation time for solutions of very low polymer concentration by doing an extrapolation from the relaxation time obtained using the extensional rheometer (CaBER) for the highest concentrations (400 and 250 $\mathrm{ppm})$ to the lowest concentration (50 ppm) based on the assumption that $W i_{c r}$ is essentially independent of the polymer concentration. This assumption is expected to hold as long as inertial effects are not important. In this case, as the critical Weissenberg number is $W i_{c r} \approx 4$, the relaxation time for the 50 ppm solution can be estimated using Equation 2:

$$
W i_{c r}=\lambda \dot{\epsilon} \approx \frac{\lambda\left(U_{2}-U_{1}\right)}{L_{c}}=\lambda \frac{Q_{c r}}{h L_{c}}\left(\frac{1}{D_{2}}-\frac{1}{D_{1}}\right)
$$

obtaining $\lambda \approx 5 \mathrm{~ms}$, which is similar to the value measured in the CaBER $(\lambda=4 \pm 1 \mathrm{~ms})$.

As already discussed, at high $W i$ an increase of the vortex size due to the progressive enhancement of elastic effects is observed. Moreover, in the experiments reported by McKinley et al. [33], and Sousa et al. [34] using a different aqueous polymer solution (PEO), the vortex growth upstream of the contraction presents either asymmetric and/or time-dependent behaviour 
which increases in magnitude with $W i$, while in the present case the flows are still steady and symmetric at the flow rates studied, as they are below the critical point.

This vortex growth regime in viscoelastic fluid flows in contractions has been also widely reported at the macro-scale. For instance, Alves et al. [35] used a more viscous Boger fluid to characterize the flow patterns in a square-square contraction, observing the formation of vortices upstream the contraction plane at low $D e$ (or low $W i$ ) which increased with $D e$ until the flow became chaotic-like at high flow rates. Note that these flows were also characterized by small Reynolds numbers, $R e<1$. Rothstein and McKinley [36] studied experimentally the flow of a Boger fluid in an axisymmetric contraction/expansion and they also reported an increase in the normalized pressure drop at high $D e$ which is believed to be the result of an additional resistance due to the extensional flow in the contraction (strain-hardening of the extensional viscosity). Later on, Sousa et al. [37] studied the flow of a Boger fluid through square-square contractions with different contraction ratios, and identified a number of distinct flow type regions with increasing values of De: at low $D e$ a region in which lip and corner vortices coexist followed at higher $D e$ by two distinct regions of diverging flow which are associated with vortex growth but exhibit different characteristics for low and high contraction ratios; and at large $D e$ the onset of unstable flow in which the vortex size varies periodically in time.

\subsection{Microfluidic diode}

A microfluidic diode, or rectifier, is a micro-channel with anisotropic flow resistance in both flow directions. The anisotropic behaviour can be due to 
inertial or elastic up-aft symmetry breaking effects when Newtonian or viscoelastic fluids flow in these micro-devices, respectively. Microfluidic diodes can be employed for instance in fixed geometry micro-pumps, which are commonly used in micro total analysis systems ( $\mu \mathrm{TAS}$ ) for pumping fluids $[38,39]$. The first fluidic rectifier was patented by Tesla [40] for Newtonian fluids based on inertial non-linear effects at high Re flows.

For viscoelastic fluids, Groisman and Quake [41] proposed a microfluidic device consisting of 43 triangular cavities connected in series, which exhibited a maximum diodicity $(D i)$ of about $2(D i$ is defined as the ratio between the backward, higher, and forward, lower, flow rates through the device for a given pressure drop). Later, a significant increase in the diodicity was achieved by Sousa et al. [42, 43], using microfluidic diodes made from 42 similar hyperbolic shaped cavities connected in series, using different aspect ratios. The aspect ratio $(A R)$ is defined as the ratio between the depth of the channel and its smallest width $\left(w_{c}\right)$ at the neck of the contraction (cf. Figure 7a for the geometry of a single element) and microfluidic diodes with $A R=$ $0.73,1.26$ and 1.71 were used in the experiments. The viscoelastic fluid used was an aqueous solution of PEO at a concentration of $0.1 \mathrm{wt} \%$ (Table 1). The polymer solution (PEO1000 in Figure 2) has a shear-thinning behaviour with a zero-shear rate viscosity of $\eta_{0}=7.5 \mathrm{mPa} \cdot \mathrm{s}$, an infinite shear rate viscosity of $\eta_{\infty}=3 \mathrm{mPa} \cdot \mathrm{s}$ and a relaxation time determined in the CaBER device, $\lambda$ $=73.9 \mathrm{~ms}$ (Figure $2 \mathrm{~b}$ ). The Reynolds number is defined as $R e=\rho U_{c} w_{c} / \eta_{0}$, where $U_{c}$ is the average velocity at the narrow passage with width $w_{c}$, and the Weissenberg number is defined as $W i=\lambda U_{c} /\left(w_{c} / 2\right)$.

The flows are characterized by low $R e$ and under these conditions the flow of 
the Newtonian fluid is found to be similar in both flow directions as in the limit of $R e \rightarrow 0$ due to creeping flow reversibility, hence the diodicity is one. Recirculations appear in both flow directions and grow within the hyperbolic elements as $R e$ is increased, but the flow remains symmetric relative to the centreline, as shown in Figure 7a, with no significant rectification effects.

For the viscoelastic fluid flow we observed an entirely different dynamic flow behaviour, with elasticity-induced recirculations appearing inside the hyperbolic corners, except at low flow rates (low Wi) where the flow is Newtonianlike. As the elasticity is increased, elastic instabilities appear first in the forward direction as shown in Figure $7 \mathrm{~b}$ and only at higher $W i$ do they appear in reversed flow. With the onset of the elastic instabilities, the vortices appear and disappear along time in some elements of the diode (unsteady flow). The critical Weissenberg number $\left(W i_{c r}\right)$ for the onset of these elastic instabilities in forward flow increases with the depth of the channel $(h)$ : for $A R=0.73, W i_{c r} \approx 20 ;$ for $A R=1.26, W i_{c r} \approx 25 ;$ and for $A R=1.71, W i_{c r} \approx$ 45.

Profiles of the normalized streamwise velocity measured with $\mu-P I V$ along the centreline of several consecutive elements show a large difference in the amplitude of the velocity oscillations in both flow directions, as can be seen in Figure 8. This dissimilar behaviour in the two directions is in contrast to the results for Newtonian fluids where the amplitudes of the oscillations of the velocity profiles in backward and forward flow were the same, as documented by Sousa et al. [42] and not shown here for conciseness. In the backward direction, for which the viscoelastic flow remains steady up to higher flow rates, the velocity gradient at the centreline is higher than for the forward direction 
(unsteady flow). Note that in the latter case the velocity field was averaged over a long period of time, in which the flow behaviour was varying in time due to the elastic instability and consequently, the velocity oscillations were smoothed. In those unsteady flow experiments, time-averaged measurements were made over a time scale significantly larger than the fluid relaxation time to establish the overall time-averaged flow field.

For viscoelastic fluid flow, the pressure drop measured along the microchannel also depends on the flow direction, leading to enhanced diodicity. As shown in Figure 9a, the pressure drop is higher in the forward direction, where the flow is more sensitive to elastic instabilities leading to unsteady flow at lower $W i$ than in the backward flow direction, so that the diodicity increases significantly above this first flow transition. At higher flow rates (or Wi), when the flow also becomes unsteady in the backward direction, the corresponding pressure drop ceases to differ so significantly from that in the forward direction leading to a reduction of diodicity at high $W i$. The stronger and more homogeneous extensional flow in the forward direction is responsible for the earlier onset of elastic instabilities and of enhanced diodicity $(D i)$, especially for higher aspect ratios as shown in Figure 9b. Using the hyperbolic-shaped micro-devices a maximum diodicity of about 6.4 was achieved when the effect of the bounding walls decreased, thus minimizing shear effects and enhancing the extensional flow component [42].

\subsection{Flow-focusing device}

The geometry of a flow-focusing device is similar to a cross-slot device, but the flow comprises three inlets and one outlet channel (all of width $D$, cf. Figure 4c), thus imposing an extensional flow to the central stream of the 
device, as shown in the Newtonian fluid flow patterns of Figure 10. This geometry has been used at the micro-scale for a number of applications, such as rheometry [44], fluid mixing [45], or droplet formation [46].

We investigated experimentally the flow of a Newtonian fluid, de-ionized water [47, 48], and of a viscoelastic fluid [47], which was an aqueous solution of $125 \mathrm{ppm}(\mathrm{w} / \mathrm{w})$ of PAA with $1 \%$ of $\mathrm{NaCl}$ (Table 1) resulting in a Boger-like fluid with a nearly constant viscosity of $\eta=1.31 \mathrm{mPa} \cdot \mathrm{s}$ and a relaxation time of $\lambda=12.4 \mathrm{~ms}$, measured in the CaBER device (Figure 2). For the Newtonian fluid flow, Figure 10 compares the experimental flow patterns with numerical predictions obtained at the same flow conditions showing a very close match. The Newtonian fluid flow remained symmetric relative to the horizontal centreline (cf. Figure 10) for a wide range of flow conditions $(R e \lesssim 113)$. The Reynolds number, based on the exit channel, is defined as $R e=\rho U_{3} D_{3} / \eta$, where $U_{3}$ and $D_{3}$ are the corresponding average velocity and channel width. Furthermore, the flow rate ratio $\left(F R=Q_{2} / Q_{1}\right)$ or the corresponding velocity ratio $\left(V R=V_{2} / V_{1}\right)$, defined as the ratio of the inlet average velocities in the lateral streams to the average velocity in the central inlet stream, controls the total Hencky strain experienced by the fluid in the converging region [47] (shown by separation streamlines in Figure 10).

For the viscoelastic fluid, a symmetric flow was also observed but only at sufficiently low $W i$ as shown in Figure 11a. As $W i$ is increased (defined as $\left.W i=\lambda U_{3} / D_{3}\right)$, two types of instabilities were observed [48]: a first transition in which the steady symmetric flow becomes asymmetric but remains steady (Figure 11b) and a second instability at higher $W i$ in which the steady asymmetric flow becomes unsteady (Figure 11c). 
The first transition is driven by elastic normal stresses that act along the streamlines and is entirely absent from the corresponding Newtonian fluid flow. Compared to the cross-slot flow discussed by Poole et al. [49], the main differences lie in the fact that there is no stagnation point at the centre of the geometry and that the onset of elastic driven instabilities depends not only on $W i$, but also on the velocity ratio as shown in Figure 12. For example, we observed that for very low $V R$ the flow evolves directly from steady symmetric to unsteady flow without ever going through the intermediate regime of steady asymmetric flow. This behaviour was also captured in the numeric computations [19] and was attributed to the normal stresses not being sufficiently high to trigger that intermediate transition. The transition from steady symmetric to steady asymmetric flows acts therefore as a stress relief mechanism.

\subsection{Confined cylinder}

The flow past a cylinder is a classic problem in fluid dynamics. The shallow depths typical of microfluidics bring a renewed interest into this topic, in particular on the effects of the aspect ratio. The flow of a Newtonian and a viscoelastic Boger fluid past a confined cylinder of diameter $D$ placed in the centre of a micro-channel (width $H=212 \mu \mathrm{m}$, cf. Figure $4 \mathrm{~d}$ ) was investigated to assess the effects of the blockage ratio ( $B R$, ratio between the cylinder diameter and the width of the microfluidic device) and aspect ratio $(A R$, ratio between the depth of the microfluidic device and the cylinder diameter). To characterize the Boger fluid flow we use the Weissenberg number, $W i=\lambda U /(D / 2)$ and when necessary the Reynolds number is defined 
as $R e=\rho U D / 2 \eta$. The micro-geometries were designed with different widths and depths to obtain $A R=2.0,1.0$ and 0.55 and $B R=25 \%, 50 \%$ and $75 \%$. De-ionized water was used as Newtonian fluid and the Boger fluid was an 85 wt\% aqueous solution of glycerol and PAA at a weight concentration of $200 \mathrm{ppm}$ with $1 \% \mathrm{NaCl}$ (Table 1). The Boger fluid showed a nearly constant shear viscosity of $0.152 \mathrm{~Pa} \cdot \mathrm{s}$ over a wide range of shear rates. The characteristic relaxation time of the Boger fluid measured in the CaBER device was $\lambda=86.7 \mathrm{~ms}$ at $293.2 \mathrm{~K}$ (Figure $2 \mathrm{~b}$ ).

For the Newtonian fluid flow at creeping flow conditions, there is no flow separation and the flow patterns are symmetric both upstream and downstream of the cylinder, due to creeping flow reversibility of Newtonian flows. At non-negligible Reynolds numbers, the measurements confirm the onset of flow separation downstream of the cylinder above a critical value of $R e$, in agreement with Ribeiro et al. [50]. This critical Re depends on $A R$ and $B R$ and increases with $B R$ with a more pronounced effect at higher $B R$.

Over the wide range of $W i$ investigated the following flow regimes were identified: Newtonian-like flow; flow with steady divergent streamlines; periodic flow; chaotic-like flow. This sequence of flow transitions takes place as $W i$ is increased and the critical conditions for the transitions were found to be dependent on $A R$ and $B R$. For low $W i$, regardless of $A R$ and $B R$, the flow is always symmetric upstream and downstream of the cylinder. Increasing $\mathrm{Wi}$, diverging streamlines progressively appear upstream of the cylinder. Both the critical conditions for the onset of the diverging streamlines and the intensity of this effect depend on the values of $A R$ and $B R$ : Figure 13 illustrates that for $B R=50 \%$ and $W i \approx 30$ the intensity of the diverging streamlines in- 
creases as $A R$ decreases. Moreover, we also found that for the same $W i$, the intensity of the divergent streamlines increases as $B R$ increases. Therefore, in both cases the divergent streamlines become stronger with wall confinement. As $W i$ increases further, the divergent streamlines become progressively more pronounced until an elastic instability arises upstream of the cylinder, near the forward stagnation point, leading to time-dependent flow. For the range of flow conditions tested, elastic instabilities were observed only for $B R=$ $75 \%$. Figure 14 shows the profiles of the streamwise dimensionless velocity along the centreline for the Boger fluid flow in order to illustrate the effects of: elasticity at constant $A R$ and $B R$ (Figure 14a) and of the aspect ratio for constant $W i$ and $B R$ (Figure $14 \mathrm{~b})$.

The streamwise velocity far upstream of the cylinder is similar to that of the Newtonian fluid flow, since the shear viscosity is constant and the shape of the fully-developed velocity profile is exclusively determined by the shear stress. Upstream of the cylinder the Boger fluid flow is independent of the elasticity of the fluid (cf. Figure 14a), but it naturally depends on the aspect ratio of the inlet channel (cf. Figure 14b) with the maximum velocity of the fully-developed profile occurring for $A R=2$ (square channel). Downstream of the cylinder, the length required for flow redevelopment increases progressively with $W i$ (cf. Figure 14a),regardless of $A R$ and BR. Figure 14b shows that as geometric confinement increases the spatial influence of the cylinder grows: the flow deceleration upstream of the cylinder starts slightly earlier and the downstream flow redevelopment length increases. 


\subsection{Microfluidic analogues of a porous medium}

Over the last decades, the flow of non-Newtonian fluids through porous media has gained significant importance in applications related to the petroleum industry, as in enhanced oil recovery, among others. The combination of the non-linear properties of those fluids with the meandering paths within the media, thus combining shear and extensional flow features, results in a complex fluid mechanics problem. Moreover, the porous media are typically opaque thus preventing the use of optical techniques. In order to obtain a better insight about the dynamics of viscoelastic fluid flows through porous media we designed simple micro-channel analogues consisting of a sequence of contractions/expansions arranged in symmetric and asymmetric configurations as shown in Figure 4e [51]. Dilute aqueous solutions of PAA at 50 and 125ppm (Table 1 and Figure 2) were used as model viscoelastic fluids in the porous media analogues, as well as in the real porous media consisting of unconsolidated packed beds (beach sand).

Measurements with de-ionized water flowing through symmetric and asymmetric micro-channels showed a linear variation of the streamwise pressure gradient with the flow rate under laminar flow conditions. Using Darcy's law it was possible to relate the modified permeabilities of the micro-channels with the permeability of the porous medium while obtaining the porosity of the porous medium and the Sauter's mean diameter $\left(x_{32}\right)$ of the particles as described in [51]. It was found that the bed of beach sand with an average particle size of $x_{32} \approx 400 \mu \mathrm{m}$ and a porosity $\epsilon=0.36$ is characterized by a modified permeability of $k_{P M}^{\prime}=2.8 \times 10^{-10} \mathrm{~m}^{2}$, which is similar to the permeability of the analogue micro-channel. The results plotted in Figure 15a 
show that both micro-channel configurations mimic well the pressure dropflow rate variation for Newtonian fluids when the equivalent particle size is selected properly, corroborating in this way and in terms of these flow properties at least, that both simplified microfluidic systems are reasonably good analogues of a real porous medium when a Newtonian fluid is used.

For viscoelastic fluids the situation is somehow different (Figure 15b). Here, the Weissenberg number is defined as $W i=\lambda \frac{U_{i}}{d_{p M C}}$, where $\lambda$ is the relaxation time of the fluid, $U_{i}$ is the velocity in the contraction sections and $d_{p M C}$ is the equivalent particle size of the analogous porous medium. At low $W i$, the pressure drop measured experimentally across the bed varies linearly with flow rate, but above a critical Weissenberg number $\left(W i_{c r}\right)$, a larger slope in the pressure gradient curve is observed due to the onset of elastic instabilities in both the asymmetric micro-channel and real porous medium (critical conditions depend on the geometry, see Figure 16). According to the PakdelMcKinley criterion $[14,15,17]$, since the flow in the asymmetric configuration is characterized by more marked streamline curvatures, the onset of elastic instabilities is expected to occur at lower $W i$ as found in the experiments. The symmetric configuration was found to reproduce well the flow of viscoelastic fluids at low $W i$, whereas the asymmetric configuration provides better results at high $W i$ (Figure 15b). In this way, both micro-geometries seem to be complementary and could be interesting tools to obtain a better insight about the flow of viscoelastic fluids through a porous medium.

Finally, on the pursuit of analysing the elastic instabilities without the influence of shear thinning effects, we have also studied the flow of low-viscosity Boger fluids (aqueous solutions of 50 and 125 ppm of PAA with $1 \%$ of $\mathrm{NaCl}$, 
see Table 1 and Figure 2 cf. [32]), through the same porous medium. Above a critical flow rate, there is a large increase in the slope of the pressure gradient curve (cf. Figure 17) due to the onset of elastic effects as discussed previously. Then further increasing the flow rate, a more dramatic rise in the slope of the pressure gradient vs. flow rate curve arises, i.e. showing an unexpected very large third slope (Figure 17). This third slope could not be reproduced in any of the microfluidic analogues of a porous medium and was found to be related to the blocking of the porous medium due to the apparent formation of a gel under these very extreme flow conditions [52].

\section{Conclusions}

The small size of microfluidic devices implies that often their flows are characterized by low Reynolds numbers especially when the fluids contain additives that increase their viscosity. In addition, such additives usually impart non-Newtonian behaviour and in particular elasticity to the fluid, leading to high Weissenberg number flows in micro-channels. These are ultimately responsible for the appearance of elastic instabilities at low or even negligible Reynolds numbers. This work summarizes five experimental investigations on pressure driven flows in micro-geometries that generate flows with a non-negligible component of extensional deformation and where the onset of various elastic instabilities are observed.

In all the symmetric geometries used the flows are Newtonian-like, steady and symmetric at low Weissenberg numbers. However, upon increasing the Weissenberg number elastic instabilities set in and the flow either becomes steady asymmetric or unsteady. When a transition to steady asymmetric 
flow takes place there is invariably a second elastic transition to unsteady flow at a higher Weissenberg number. The path of transitions also depends on other relevant dimensionless numbers specific to each geometric flow: for flow-focusing it is possible to evolve directly from steady symmetric to unsteady flow, without ever going through the steady asymmetric flow, for some ranges of the velocity ratio and Reynolds number. Presumably, the different transitions are associated with different mechanisms involving elastic normal stresses. In some cases extremely high normal stresses lead to a specific flow feature which is not observed under weaker flow conditions. In other cases the curved streamlines are unable to sustain the normal stresses acting along those streamlines as described by the Pakdel-McKinley criterion [14, 15, 17], and the flow becomes unstable.

These elastic instabilities have both advantages and drawbacks in practical terms. When the objective is micro-mixing or high rates of heat and mass transfer, these low Reynolds number chaotic-like flows of elastic fluids provide a useful solution which is not available if the fluids are Newtonian (a similar situation here would require higher Reynolds numbers for chaotic advection to emerge). However, if the objective is to minimize or delay the existence of unstable flows, as when operating with micro-rheometers, then ideally there should be no and these transitions impose operational limits.

As shown in the examples described, new phenomena arise at the micro-scale when fluids are non-Newtonian and these flows have interesting features especially in combination with other unusual conditions not described here, such as in the presence of electrokinetic effects or of gradients in surface tension made possible by new developments and advances in micro-manufacturing 
and surface coating methods. The popularity of micro-flow systems requires the miniaturization of flow forcing methods and the use of electric and magnetic forcing mechanisms will certainly become more common as an alternative to pressure driven flow. The combination of these methods with fluids made from complex additives, will certainly provide an opportunity to explore new challenging phenomena. Already good examples are the combinations of microfluidics and optics (optofluidics) and of microfluidics and acoustics (acousto-fluidics), for instance for particle mixing or separation in micro-channels.

\section{Acknowledgements}

The authors acknowledge the funding from FCT, COMPETE and FEDER

through various projects and scholarships throughout the years: PTDC/EQU-

FTT/70727/2006, PTDC/EME-MFE/114322/2009, PTDC/EME-MFE/99109/2008

and PTDC/EQU-FTT/118716/2010, SFRH/BPD/69663/2010, SFRH/BPD/69664/2010,

SFRH/BPD/75258/2010, SFRH/BD/44737/2008, IF/00148/2013 and IF/00190/2013. 
Table 1: Formulations of the viscoelastic fluids used.

\begin{tabular}{l|cc|cc|c|c}
\hline \hline \multirow{2}{*}{$a$} & \multicolumn{2}{|c|}{ Polymer } & \multicolumn{2}{c|}{ Solvent } & \\
& PAA & PEO & Water & Glycerol & $\mathrm{NaCl}$ & $\lambda$ \\
& {$[\mathrm{ppm}]$} & {$[\mathrm{ppm}]$} & {$[\%]$} & {$[\%]$} & {$[\%]$} & {$[\mathrm{ms}]$} \\
\hline \hline PAAs50 & 50 & 0 & 100 & 0 & 1 & $4 \pm 1$ \\
PAA50 & 50 & 0 & 100 & 0 & 0 & $54 \pm 1$ \\
PAAs125 & 125 & 0 & 100 & 0 & 1 & $10 \pm 2$ \\
PAA125 & 125 & 0 & 100 & 0 & 0 & $129 \pm 1$ \\
PAAsg200 & 200 & 0 & 85 & 15 & 1 & $87 \pm 1$ \\
PAAs250 & 250 & 0 & 100 & 0 & 1 & $18 \pm 2$ \\
PAAs400 & 400 & 0 & 100 & 0 & 1 & $29 \pm 2$ \\
PEO1000 & 0 & 1000 & 100 & 0 & 0 & $74 \pm 1$ \\
\hline \hline
\end{tabular}

${ }^{a}$ PAA: Polyacrylamide; PAAs: PAA with $\mathrm{NaCl}$ in water; PAAsg: PAA in water with NaCL and glycerol; PEO: Polyethylene oxide 
Table 2: Dimensions of the micro-channels used in the works here reviewed (see Figure 4).

\begin{tabular}{|c|c|c|c|c|c|c|}
\hline Hyperbolic contraction & $h[\mu \mathrm{m}]$ & $D_{1}[\mu \mathrm{m}]$ & $D_{2}[\mu \mathrm{m}]$ & $L_{c}[\mu \mathrm{m}]$ & & \\
\hline (Fig.4a) & 45 & 400 & 54 & 128 & & \\
\hline & $A R[-]$ & $h[\mu \mathrm{m}]$ & $D[\mu \mathrm{m}]$ & $w_{c}[\mu \mathrm{m}]$ & $L[\mu \mathrm{m}]$ & \\
\hline Microfluidic diode & 0.73 & 46 & 326 & 63 & 128 & \\
\hline \multirow[t]{2}{*}{ (Fig.4b) } & 1.26 & 88 & 326 & 70 & 128 & \\
\hline & 1.71 & 120 & 326 & 70 & 128 & \\
\hline Flow focusing & $h[\mu \mathrm{m}]$ & $D[\mu \mathrm{m}]$ & & & & \\
\hline \multirow[t]{5}{*}{ Fig.4c } & 100 & 100 & & & & \\
\hline & $A R[-]$ & $B R[\%]$ & $h[\mu \mathrm{m}]$ & $D[\mu \mathrm{m}]$ & $H[\mu \mathrm{m}]$ & \\
\hline & 0.37 & 75 & 58 & 158 & 212 & \\
\hline & 0.55 & 55 & 58 & 105 & 212 & \\
\hline & 1.1 & 25 & 58 & 55 & 212 & \\
\hline Confined cylinder & 0.66 & 75 & 105 & 158 & 212 & \\
\hline \multirow[t]{7}{*}{ (Fig.4d) } & 1.0 & 50 & 105 & 105 & 212 & \\
\hline & 1.9 & 25 & 105 & 55 & 212 & \\
\hline & 1.3 & 75 & 213 & 158 & 212 & \\
\hline & 2.0 & 50 & 213 & 105 & 212 & \\
\hline & 3.9 & 25 & 213 & 55 & 212 & \\
\hline & $W_{1}[\mu \mathrm{m}]$ & $W_{2}[\mu \mathrm{m}]$ & $W_{c}[\mu \mathrm{m}]$ & $L_{1}[\mu \mathrm{m}]$ & $L_{2}[\mu \mathrm{m}]$ & $h[\mu \mathrm{m}]$ \\
\hline & \multicolumn{6}{|c|}{ Symmetric arrangement } \\
\hline \multirow{3}{*}{$\begin{array}{l}\text { Analogues of a porous medium } \\
\qquad \text { (Fig.4e) }\end{array}$} & 108 & 40 & 40 & 106 & 31 & 103 \\
\hline & \multicolumn{6}{|c|}{ Asymmetric arrangement } \\
\hline & 108 & 72 & 52 & 106 & 31 & 103 \\
\hline
\end{tabular}


Figure 1: Illustration of the onset of an elastic instability from instantaneous flow patterns of a PEO solution in a 50:50 water/glycerol mixture flowing through a serpentine channel $\left(M_{\text {crit }} \approx 0.68\right)$. From top to bottom: stable flow $(W i=0.22)$; slightly unstable flow, close to the onset of elastic instability $(W i=0.24) ;$ and unstable flow $(W i=0.25)$. Reproduced with permission from Zilz et al. [20]. 


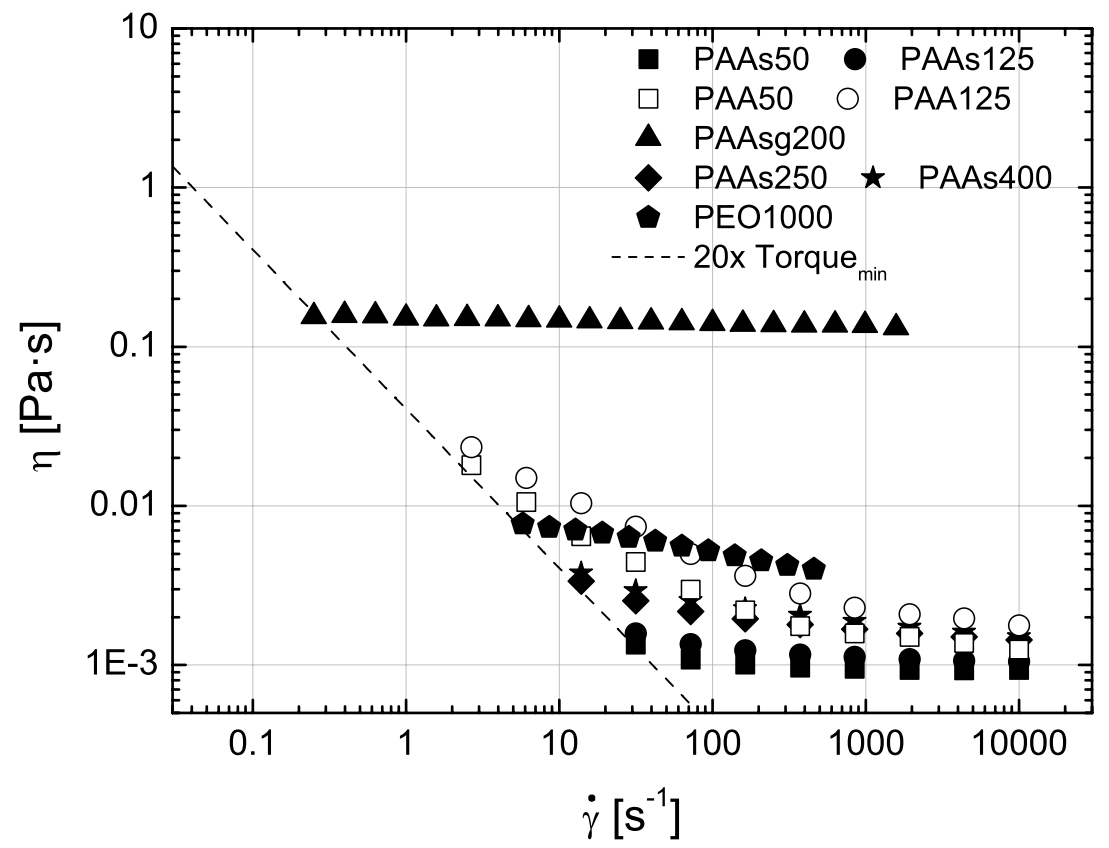

(a)

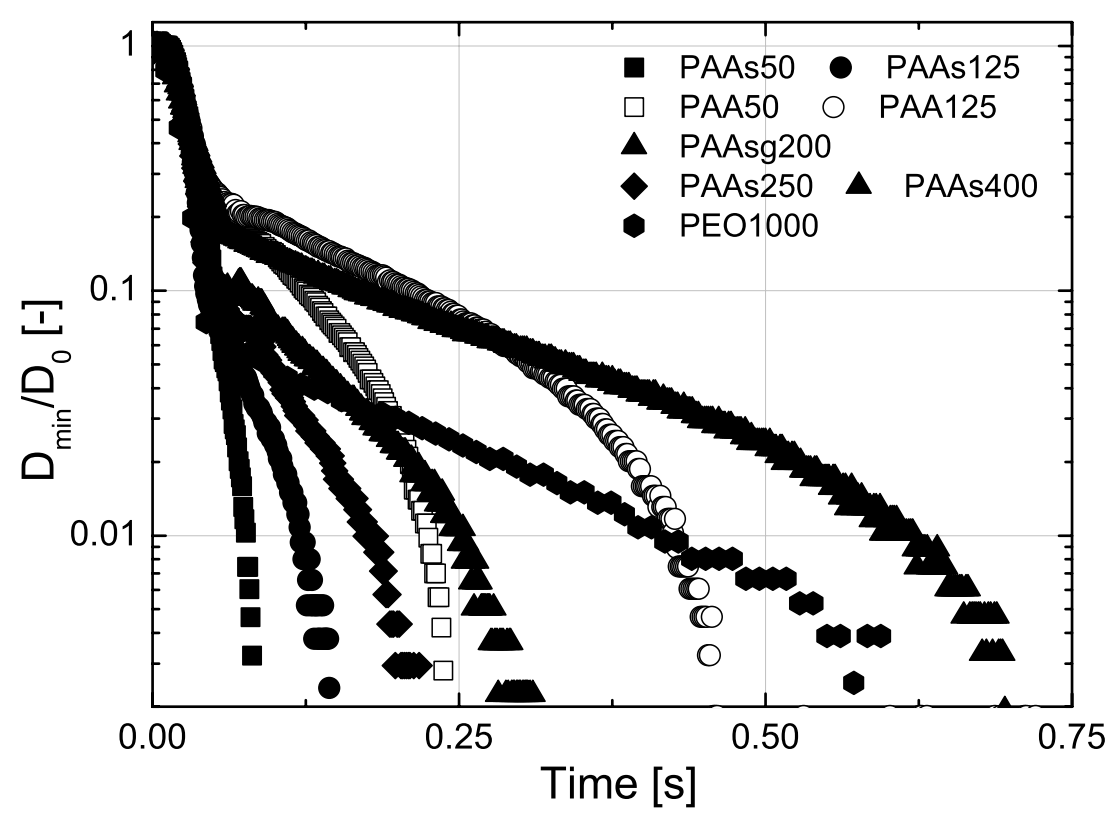

29

(b)

Figure 2: Rheological characterization of the viscoelastic fluids at $20^{\circ} \mathrm{C}$ : (a) flow curves in steady shear flow and (b) time evolution of the normalized diameter measured in the CaBER device. 
Figure 3: PDMS device fabrication procedure. (a) Cross-section of the SU-8 mould with a positive relief. (b) The mixture of oligomer and curing agent is poured onto the SU-8 mould (thick layer) and placed in the oven to cure for 20 minutes. (c) After curing, the PDMS thick layer is removed from the SU-8 mould and access ports are created. (d) The PDMS layer containing the channel structure is bonded to the glass slide covered with a thin layer of PDMS and placed in the oven to further seal the channel. 

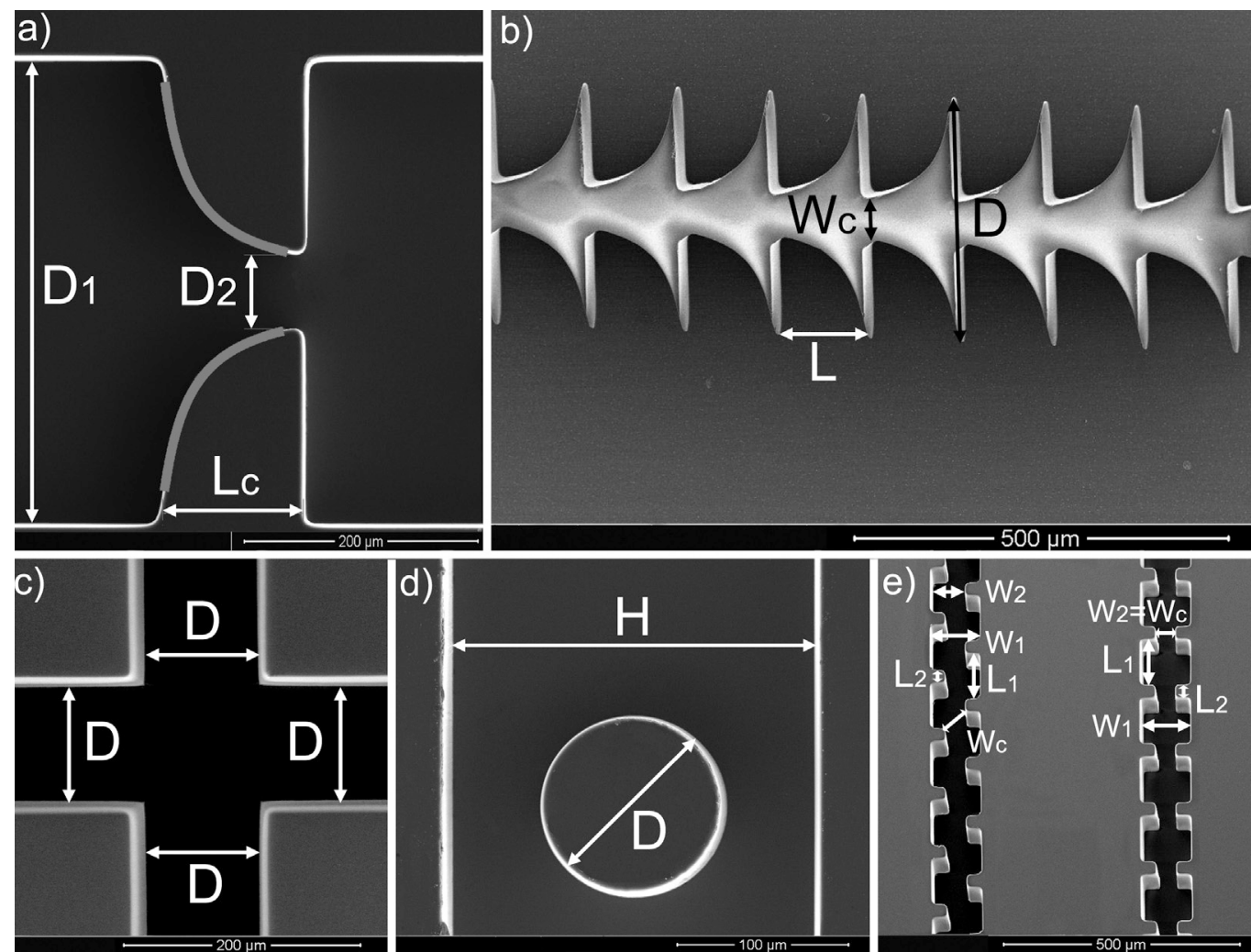

Figure 4: Scanning Electron Microscopy images of the five planar microchannels fabricated from high quality SU-8 photoresist moulds obtained by means of a high-resolution chrome mask using standard soft lithography: (a) hyperbolic contraction and sudden expansion; (b) microfluidic diode; (c) cross-slot device (to be used as a flow focusing micro-device); (d) confined cylinder; and (e) simplified microfluidic analogues of a porous medium. See Table 2 for dimensions. 


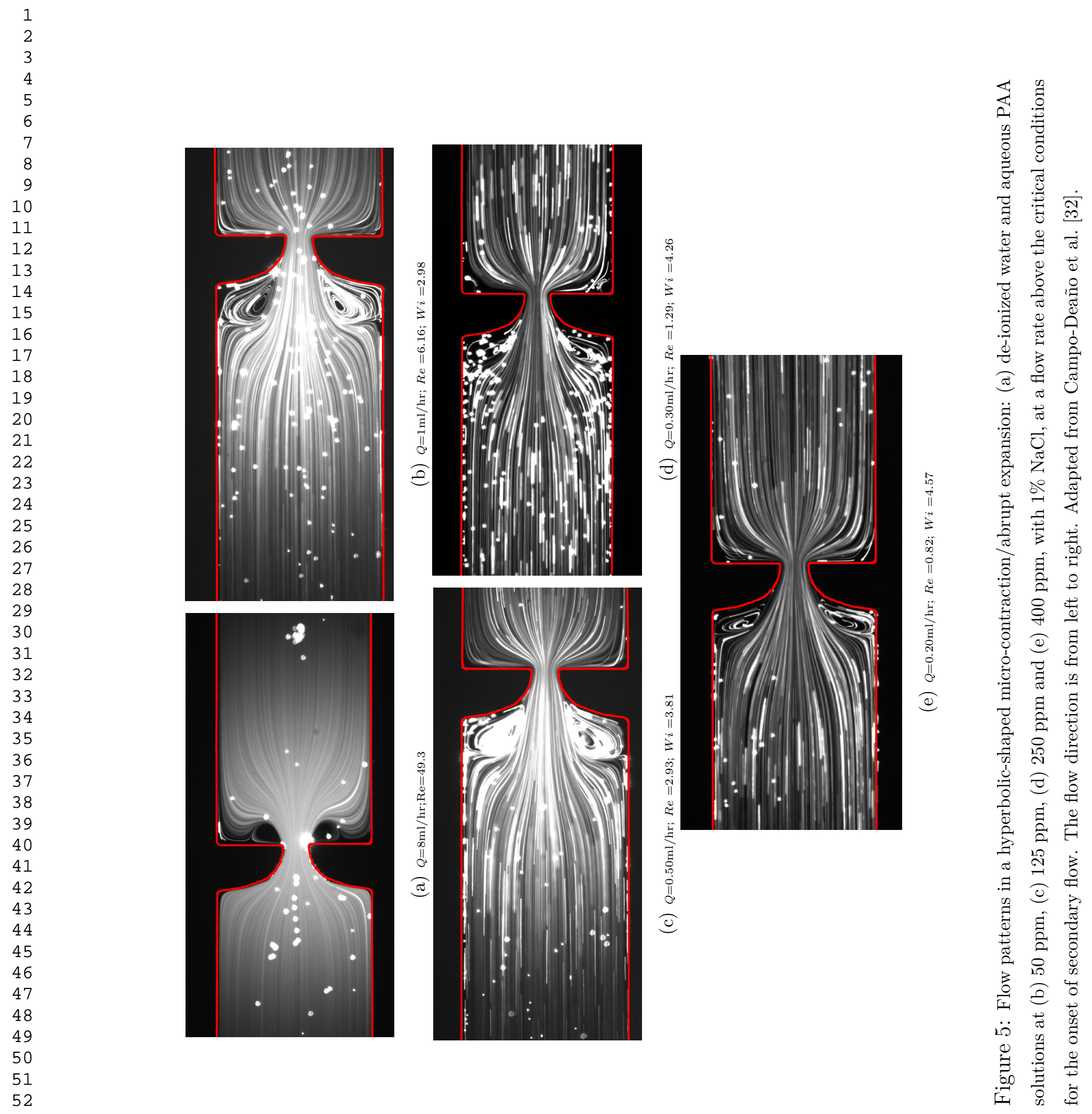




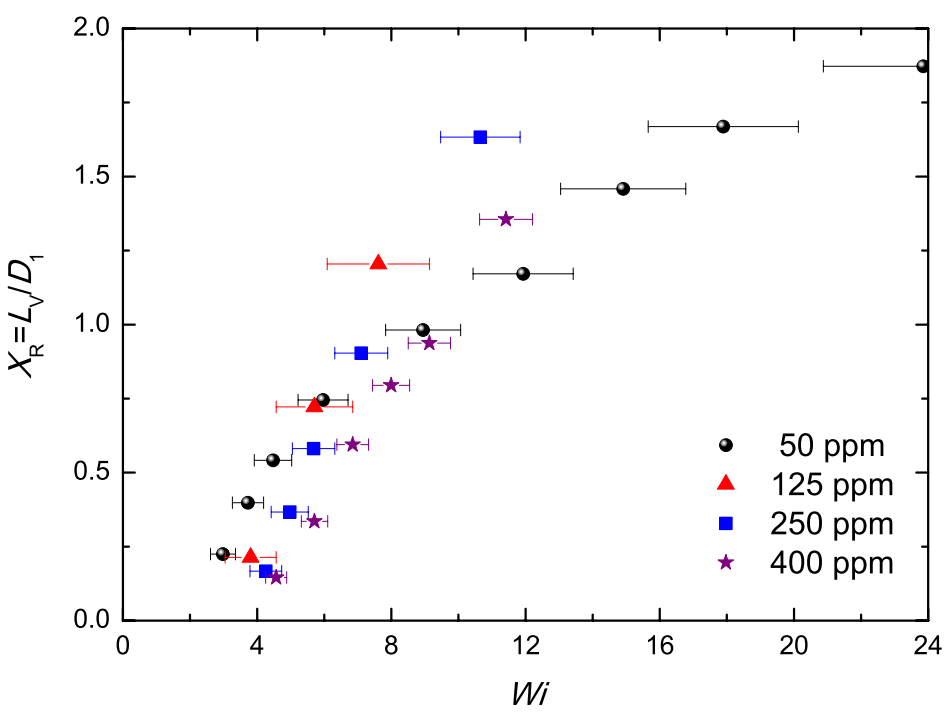

Figure 6: Effect of Weissenberg number on the dimensionless vortex length in the steady symmetric regime for the 50, 125, 250 and $400 \mathrm{ppm}$ PAA aqueous solutions with $1 \% \mathrm{NaCl}$ flowing through a hyperbolic-shaped microcontraction. Adapted from Campo-Deaño et al. [32]. 


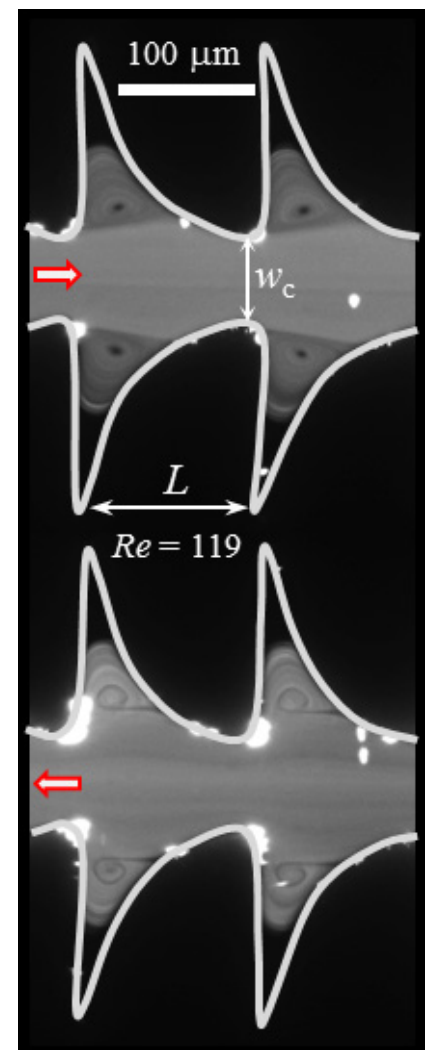

(a)

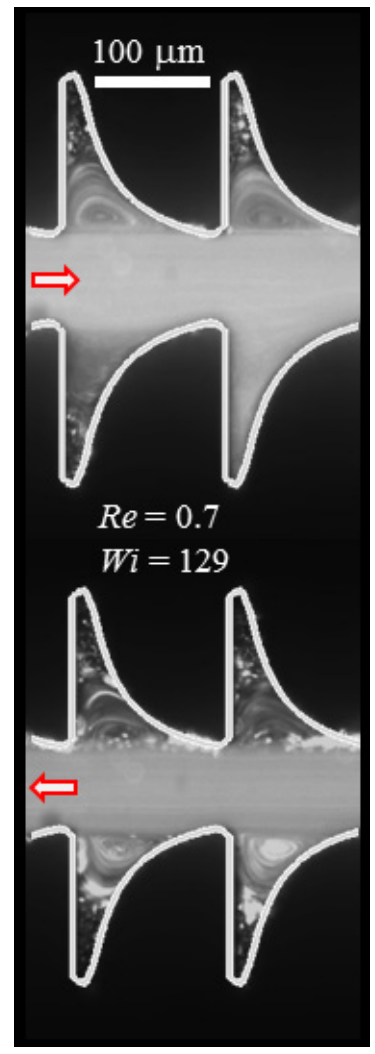

(b)

Figure 7: (a) Newtonian fluid flow patterns in a hyperbolic diode with $A R=0.73$. Adapted from Sousa et al. [43]. (b) Viscoelastic fluid flow patterns in a microfluidic diode with $A R=1.26$. Adapted from Sousa et al. [42]. 


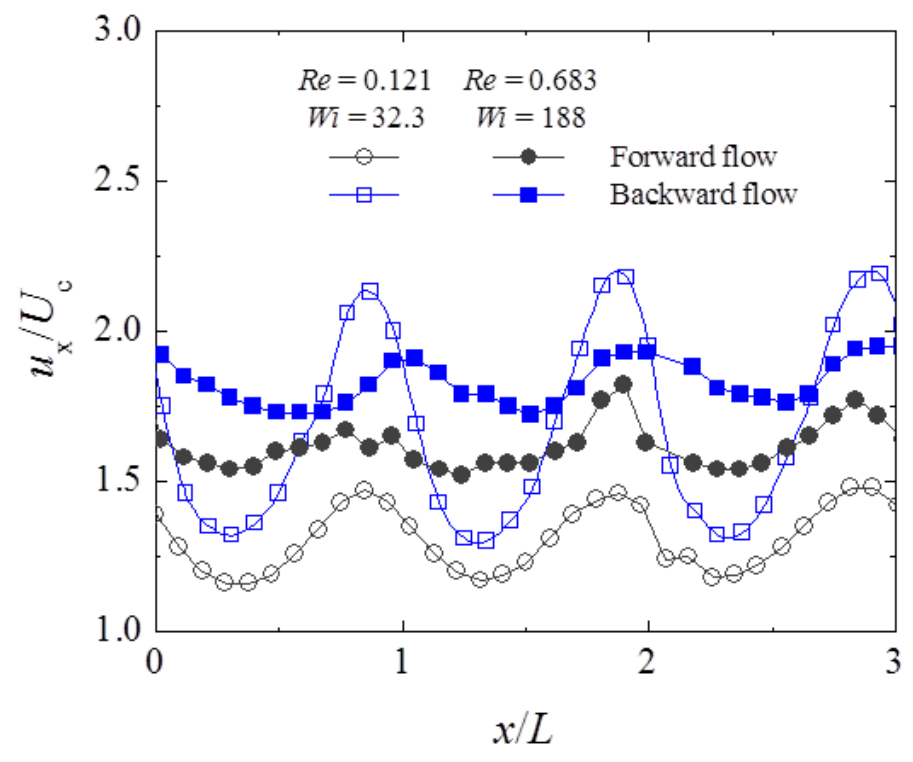

Figure 8: Dimensionless axial velocity profiles along the centreline of the microfluidic diode, $A R=0.73$, for viscoelastic fluid flow under two different flow rates. The streamwise velocity component $\left(u_{x}\right)$ is normalised with the bulk velocity at the contraction throat $\left(U_{c}\right)$. 


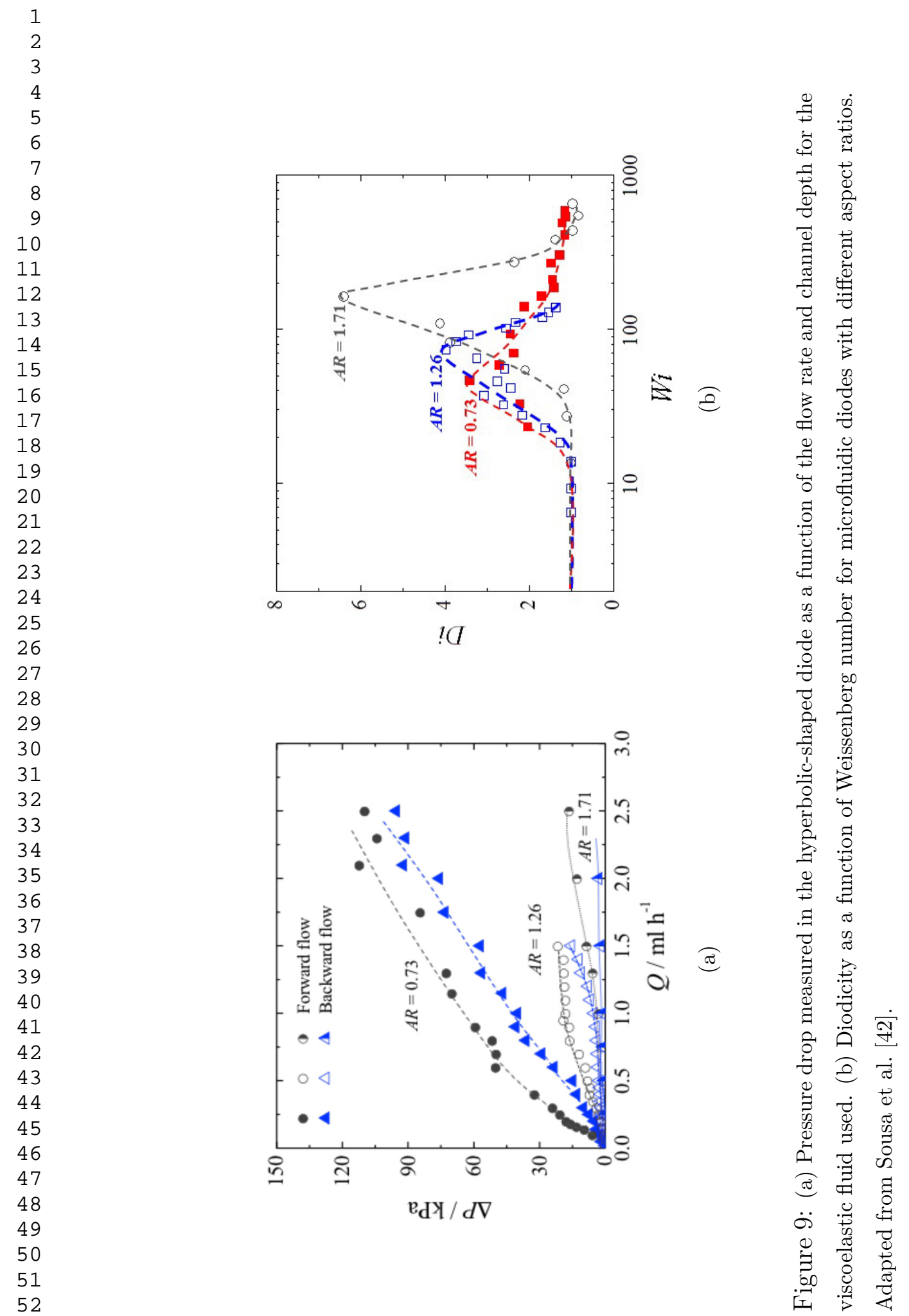

53

54

55

56

57

58

59

60

61

62

63 
Figure 10: Experimental (micrograph) and numerical (red solid lines) flow patterns obtained for Newtonian fluid flow in a flow-focusing micro-device, $Q_{1}=Q_{2}=0.3 \mathrm{ml} \mathrm{h}^{-1}, V R=1$ and $R e=2.8$. The separation streamlines are highlighted by white dashed lines and arrows indicate the flow direction. Adapted from Oliveira et al. [47]. 


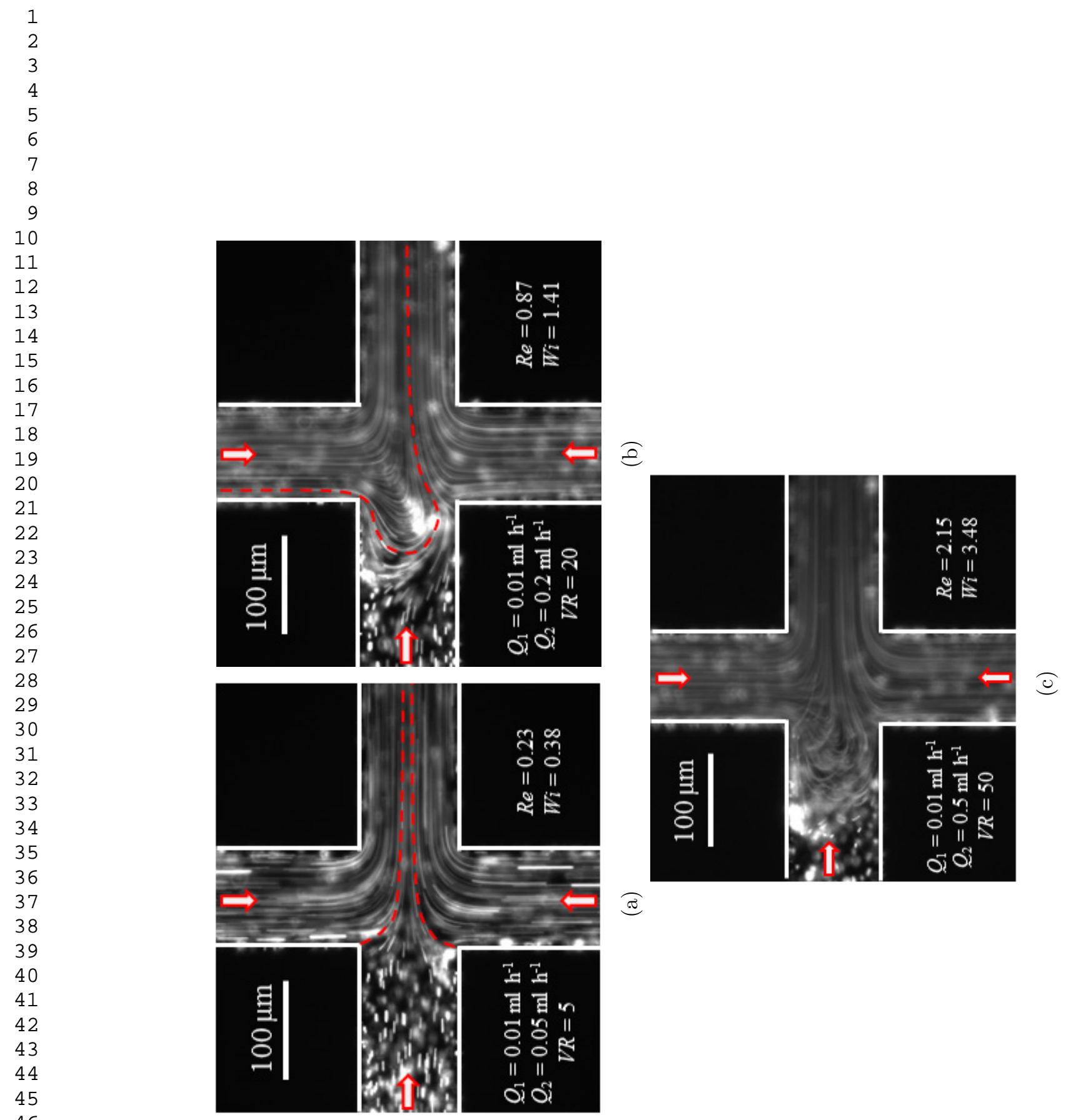




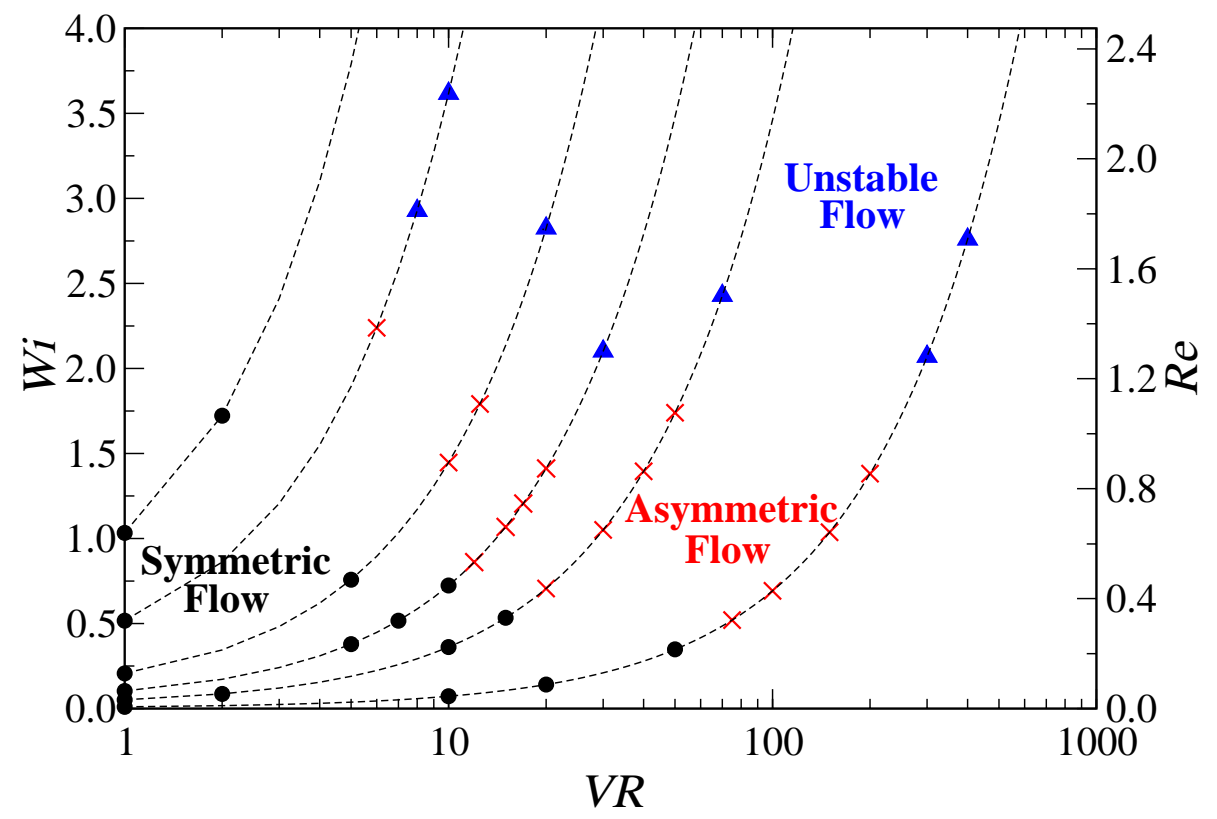

Figure 12: Flow classification map in the $W i$ - $V R$ domain for the flow-focusing micro-device. The circles indicate symmetric flow, the crosses steady asymmetric flow and the triangles unsteady flow. 
Figure 13: Flow patterns obtained for the Boger fluid flow around a cylinder for $B R=50 \%$ as a function of $A R$ under similar flow conditions: a) $A R=$ 2.0, $W i=30.5, R e=7.8 \times 10^{-3}$; b) $A R=1.0, W i=30.9, R e=7.9 \times 10^{-3}$; c) $A R=0.55, W i=29.9, R e=7.6 \times 10^{-3}$. Arrows indicate the flow direction. 


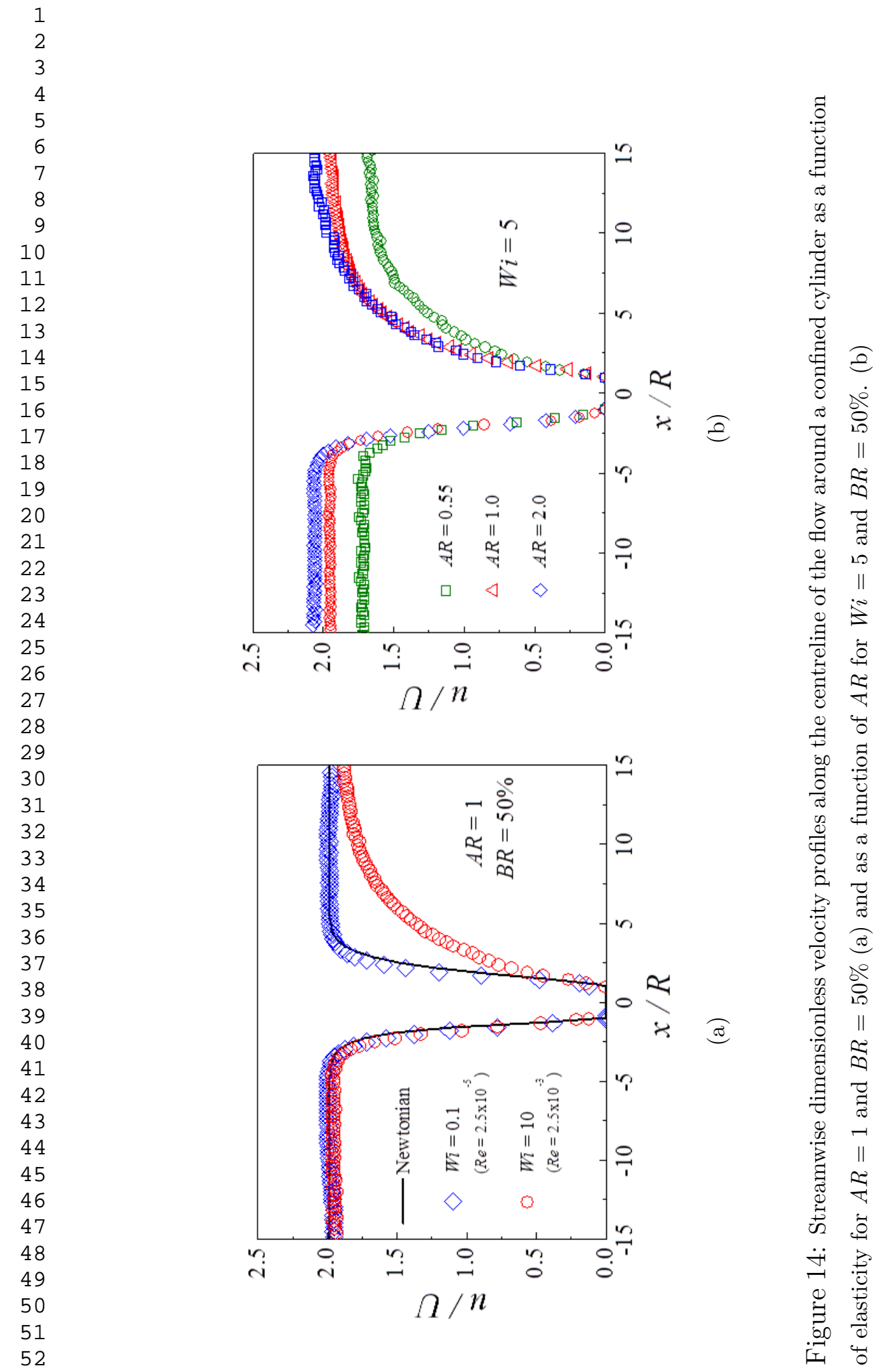

53

54

55

56

57

58

59

60

61

62

63

64

65 


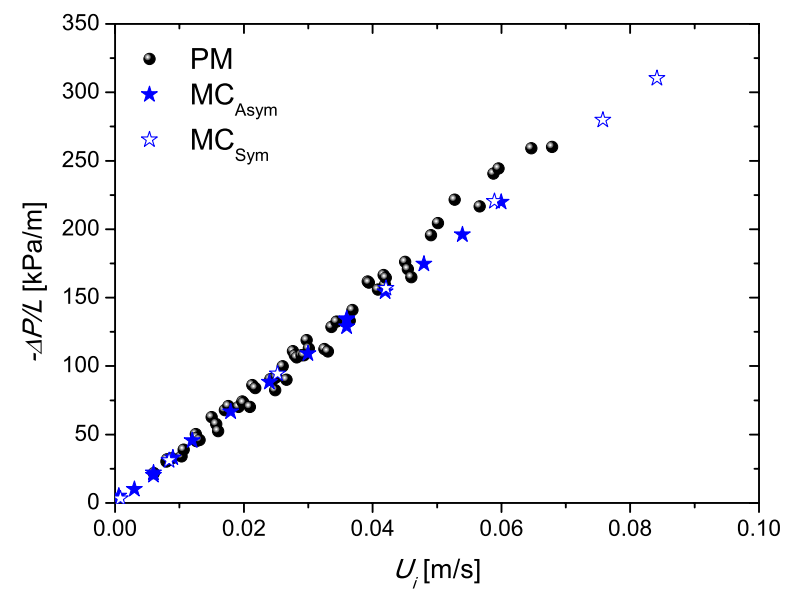

(a)

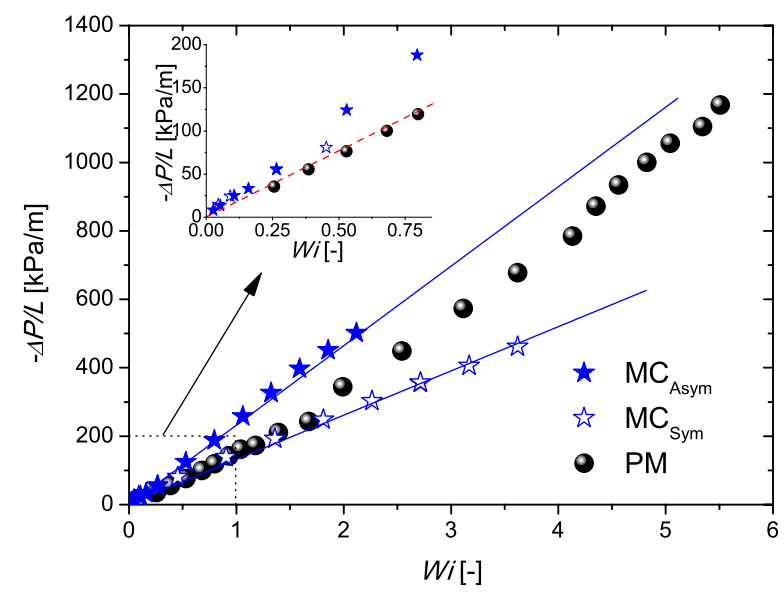

(b)

Figure 15: Average pressure drop gradient as a function of the interstitial velocity for the flow of (a) de-ionized water through the microfluidic analogues of a porous medium $\left(\mathrm{MC}_{\text {Asym }}, \mathrm{MC}_{\text {Sym }}\right)$ and the porous sand bed (PM); (b) an aqueous solution of PAA at 125ppm through the microfluidic analogues of a porous medium $\left(\mathrm{MC}_{\text {Asym }}, \mathrm{MC}_{\text {Sym }}\right)$ and the porous sand bed (PM). Adapted from Galindo-Rosales et al. [51]. $\quad 42$ 

Adapted from Galindo-Rosales et al. [51]. 


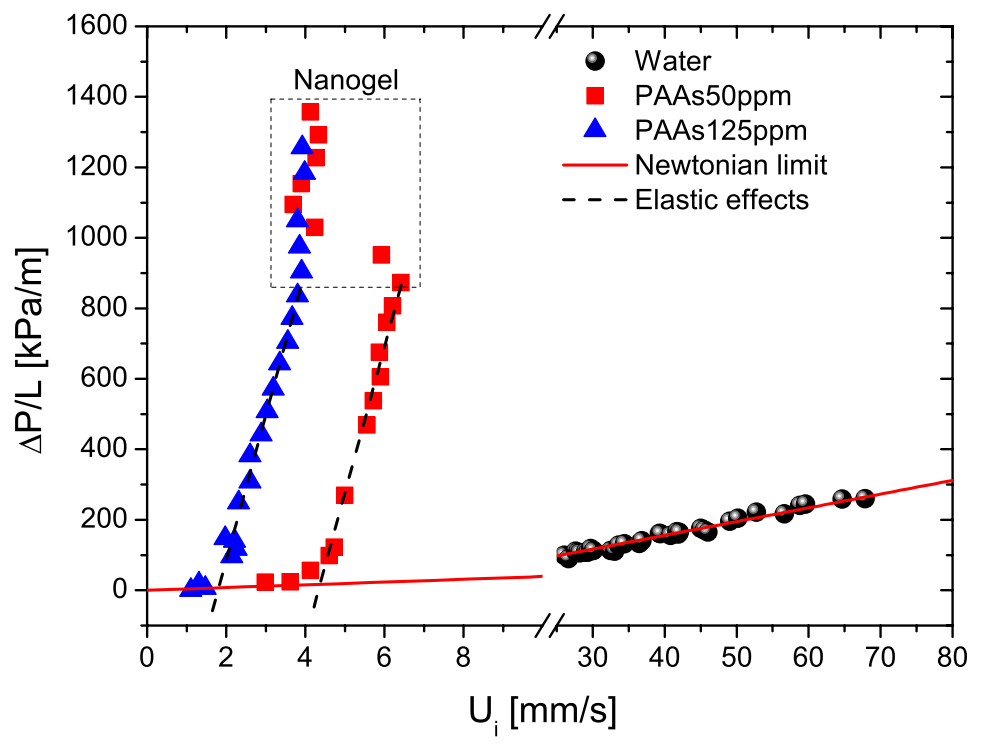

Figure 17: Variation of the pressure gradient with the interstitial velocity for the flow of low viscosity Boger fluids through a porous medium made of sand of $400 \mu \mathrm{m}$ particles (Sauter mean diameter). Adapted from Campo-Deaño et al. [52]. 


\section{References}

[1] T. Bayraktar, S. B. Pidugu, Characterization of liquid flows in microfluidic systems, International Journal of Heat and Mass Transfer 49 (2006) $815-824$.

[2] G. Karniadakis, A. Beskok, N. Aluru, Microflows and Nanoflows: Fundamentals and Simulation, Springer, New York, 2005.

[3] A. D. Stroock, S. K. W. Dertinger, A. Adjari, I. Mezic, H. A. Stone, G. M. Whitesides, Chaotic mixer for microchannels, Science 295 (2002) 647-651.

[4] R. G. Larson, The structure and rheology of complex fluids, Oxford University Press, New York, 1999.

[5] R. J. Poole, The Deborah and Weissenberg numbers, Rheology Bulletin 53 (2012) 32-39.

[6] J. M. Dealy, Weissenberg and Deborah numbers Ü their definition and use, Rheology Bulletin 79(2) (2010) 14-18.

[7] T. M. Squires, S. R. Quake, Microfluidics: fluid physics at the nanoliter scale, Reviews of Modern Pphysics 77 (2005) 977-1026.

[8] M. Brust, C. Schaefer, R. Doerr, L. Pan, M. Garcia, P. E. Arratia, C. Wagner, Rheology of human blood plasma: Viscoelastic versus newtonian behavior, Physical Review Letters 110 (2013) 078305. 
[9] L. Campo-Deaño, R. P. A. Dullens, D. G. L. A. Aarts, F. T. Pinho, M. S. N. Oliveira, Viscoelasticity of blood and viscoelastic blood analogues for use in polydymethylsiloxane in vitro models of the circulatory system, Biomicrofluidics 7 (2013) 034102.

[10] P. C. Sousa, J. Carneiro, R. Vaz, A. Cerejo, F. T. Pinho, M. A. Alves, M. S. N. Oliveira, Shear viscosity and nonlinear behaviour of whole blood under large amplitude oscillatory shear, Biorheology 50 (2013) 269-282.

[11] E. S. G. Shaqfeh, Purely elastic instabilities in viscometric flows, Annual Reviews in Fluid Mechanics 28 (1996) 129-186.

[12] J. A. Pathak, D. Ross, K. B. Migler, Elastic flow instability, curved streamlines, and mixing in microfluidic flows, Physics of Fluids 16 (2004) 4028-4035.

[13] R. G. Larson, E. S. G. Shaqfeh, S. J. Muller, A purely elastic instability in taylor-couette flow, Journal of non-Newtonian Fluid Mechanics 218 (1990) 573-600.

[14] P. Pakdel, G. H. McKinley, Elastic instabilities and curved streamlines, Physical Review Letters 77 (1996) 2459-2462.

[15] G. H. McKinley, P. Pakdel, A. Oztekin, Rheological and geometric scaling of purely elastic flow instabilities, Journal of Non-Newtonian Fluid Mechanics 67 (1996) 19-47.

[16] J. Soulages, M. S. N. Oliveira, P. C. Sousa, M. Alves, G. McKinley, Investigating the stability of viscoelastic stagnation flows in t-shaped 
microchannels, Journal of non-Newtonian Fluid Mechanics 163 (2009) 9-24.

[17] P. Pakdel, G. McKinley, Cavity flows of elastic liquids: purely elastic instabilities, Physics of Fluids 10(5) (1998) 1058-1070.

[18] M. A. Alves, R. J. Poole, Divergent flow in contractions, Journal of Non-Newtonian Fluid Mechanics 144 (2007) 140-148.

[19] M. S. N. Oliveira, F. T. Pinho, R. J. Poole, P. J. Oliveira, M. A. Alves, Purely elastic flow asymmetries in flow-focusing devices, Journal of nonNewtonian Fluid Mechanics 160 (2009) 31-39.

[20] J. Zilz, R. J. Poole, M. A. Alves, D. Bartolo, A. Lindner, Geometric scaling of a purely elastic flow instability in serpentine channels, Journal of Fluid Mechanics 712 (2012) 203-218.

[21] M. S. N. Oliveira, M. A. Alves, F. T. Pinho, Microfluidic flow of viscoelastic fluids, in: R. Grigoriev (Ed.), Transport and mixing in laminar flows: From microfluidics to oceanic currents, Wiley-VCH Verlag GmbH \& Co. KGaA, Weinheim, Germany, 2011, pp. 131-174.

[22] C. Tropea, A. L. Yarin, J. F. Foss (Eds.), Springer Handbook of Experimental Fluid Mechanics, Springer Berlin Heidelberg, Germany, 2007.

[23] A. Zell, S. Gier, S. Rafai, C. Wagner, Is there a relation between the relaxation time measured in caber experiments and the first normal stress coefficient?, Journal of non-Newtonian Fluid Mechanics 165 (2010) 1265-1274. 
[24] V. Kantsler, V. Steinberg, Transition to tumbling and two regimes of tumbling motion of a vesicle in shear flow, Physical Review Letters 96 (2006) 036001.

[25] Y. Liu, Y. Jun, V. Steinberg, Concentration dependence of the longest relaxation times of dilute and semi-dilute polymer solutions, Journal of Rheology 53(5) (2009) 1069-1085.

[26] A. Aitkadi, P. Carreau, G. Chauveteau, Rheological properties of partially hydrolyzed polyacrylamide solutions, Journal of Rheology 31 (1987) 537-561.

[27] Y. Xia, G. M. Whitesides, Soft lithography, Annual Review of Materials Science 28 (1998) 153-184.

[28] J. C. McDonald, D. C. Duffy, J. R. Anderson, D. T. Chiu, H. K. Wu, O. J. A. Schueller, G. M. Whitesides, Fabrication of microfluidic systems in poly(dimethylsiloxane), Electrophoresis 21 (2000) 27-40.

[29] D. F. James, Flow in a converging channel at moderate reynolds number, AIChE Journal 37 (1991) 59-64.

[30] M. S. N. Oliveira, M. A. Alves, F. T. Pinho, G. H. McKinley, Viscous flow through microfabricated hyperbolic contractions, Experiments in Fluids 43 (2007) 437-451.

[31] D. V. Boger, A highly elastic constant-viscosity fluid, Journal of NonNewtonian Fluid Mechanics 3 (1977) 87-91. 
[32] L. Campo-Deaño, F. J. Galindo-Rosales, F. T. Pinho, M. A. Alves, M. S. N. Oliveira, Flow of low viscosity Boger fluids through a microfluidic hyperbolic contraction, Journal of Non-Newtonian Fluid Mechanics 166 (2011) 1286-1296.

[33] G. H. McKinley, L. E. Rodd, M. S. N. Oliveira, J. J. Cooper-White, Extensional flows of polymer solutions in microfluidic converging/diverging geometries, Journal of Central South University Technology 14 (2007) 6-9.

[34] P. C. Sousa, F. T. Pinho, M. S. N. Oliveira, M. A. Alves, Extensional flow of blood analogue solutions in microfluidic devices, Biomicrofluidics 5 (2011) 014108.

[35] M. A. Alves, F. T. Pinho, P. J. Oliveira, Visualizations of Boger fluid flows in a 4:1 square-square contraction, AIChE Journal 51 (2005) 29082922.

[36] J. P. Rothstein, G. H. McKinley, Extensional flow of a polystyrene Boger fluid through a 4:1:4 axisymmetric contraction/expansion, Journal of Non-Newtonian Fluid Mechanics 86 (1999) 61-88.

[37] P. C. Sousa, P. M. Coelho, M. S. N. Oliveira, M. A. Alves, Three dimensional flow of Newtonian and Boger fluids in square-square contractions, Journal of Non-Newtonian Fluid Mechanics 160 (2009) 122-139.

[38] E. Stemme, G. Stemme, A valveless diffuser/nozzle-based fluid pump, Sensors and Actuators A: Physical 39 (1993) 159-167. 
[39] T. Gerlach, H. Wurmus, Working principle and performance of the dynamic micropump, Sensors and Actuators A: Physical 50 (1995) 135140.

[40] N. Tesla, Valvular conduit, US Patent 1329559., 1920.

[41] A. Groisman, S. R. Quake, A microfluidic rectifier: anisotropic flow resistance at low reynolds numbers, Physical Review Letters 92 (2004) 094501.

[42] P. C. Sousa, F. T. Pinho, M. S. N. Oliveira, M. A. Alves, High performance microfluidic rectifiers for viscoelastic fluid flow, RSC Advances 2 (2012) 920-929.

[43] P. C. Sousa, F. T. Pinho, M. S. N. Oliveira, M. A. Alves, Efficient microfluidic rectifiers for viscoelastic fluid flow, Journal of Non-newtonian Fluid Mechanics 165 (2010) 652-671.

[44] P. E. Arratia, J. P. Gollub, D. J. Durian, Polymeric filament thinning and breakup in microchannels, Physical Review E 77 (2008) 036309.

[45] K. Jensen, Chemical kinetics: smaller, faster chemistry, Nature 393 (1998) 735-737.

[46] S. L. Anna, H. C. Mayer, Microscale tipstreaming in a microfluidic flow, Physics of Fluids 18 (2006) 121512.

[47] M. S. N. Oliveira, F. T. Pinho, M. A. Alves, Divergent streamlines and free vortices in Newtonian fluid flows in microfluidic flow-focusing devices, Journal of Fluids Mechanics 711 (2012) 171-191. 
700

[48] M. S. N. Oliveira, F. T. Pinho, M. A. Alves, Extensional flow of Newtonian and Boger fluids through a flow focusing microdevice, 3rd Micro and Nano Flows Conference, Thessaloniki, Greece, 22-24th August, 2011.

[49] R. J. Poole, M. A. Alves, P. J. Oliveira, Purely-elastic flow asymmetries, Physical Review Letters 99 (2007) 164503.

[50] V. M. Ribeiro, P. M. Coelho, F. T. Pinho, M. A. Alves, Threedimensional effects in laminar flow past a confined cylinder, Chemical Engineering Science 84 (2012) 155-169.

[51] F. J. Galindo-Rosales, L. Campo-Deaño, M. S. N. Oliveira, M. A. Alves, F. T. Pinho, E. v. Bokhorst, P. J. Hamersma, Microfluidic systems for the analysis of viscoelastic fluid flow phenomena in porous media, Microfluidics and Nanofluidics 12 (2012) 485-498.

[52] L. Campo-Deaño, F. J. Galindo-Rosales, F. T. Pinho, M. A. Alves, M. S. N. Oliveira, Nanogel formation of polymer solutions flowing through porous media, Soft Matter 8 (2012) 6445-6453. 